\title{
Redesign of an Open-System Oxygen Face Mask With Mainstream Capnometer for Children
}

\author{
Natalie Napolitano MPH RRT-NPS FAARC, Akira Nishisaki MD MSCE, \\ Hayley S Buffman MPH, Jessica Leffelman, Matthew R Maltese PhD, and Vinay M Nadkarni MD
}

\begin{abstract}
BACKGROUND: Partial pressure of end-tidal carbon dioxide $\left(\mathbf{P}_{\mathrm{ETCO}_{2}}\right)$ monitoring in children is important to detect apnea or hypopnea early to intervene before hypoxemia develops. Monitoring $\mathbf{P}_{\mathrm{ETCO}_{2}}$ in children without a tracheal tube is challenging. To improve $\mathbf{P}_{\mathrm{ETCO}}$ measurement accuracy in a commercially available mask with a mainstream $\mathrm{CO}_{2}$ detector, we implemented design changes with deform-and-hold shaping technology and anterior-posterior adjustment of the expiratory gas flow cup. METHODS: Two sizes of redesigned face masks (small for 7-20 kg, medium for 10-40 kg) were evaluated. Initial bench testing used a simulator modeling a spontaneously breathing infant and child with a natural airway. An infant/child manikin head was connected to the breathing lung simulator. A mass flow controller provided expiratory $\mathrm{CO}_{2}$. Mask fit was then evaluated on healthy human subjects to identify anatomical features associated with good fit, defined as square shape capnography waveform during expiration. A 3-dimensional digital scan was used to quantify anatomical features. The gaps between face mask rims and facial surface were manually measured. RESULTS: Bench testing revealed a $\mathrm{P}_{\mathrm{ETCO}_{2}}$ difference of $3.4 \pm 1.5 \mathrm{~mm} \mathrm{Hg}$ between a measured $\mathrm{P}_{\text {ETCO }}$ by the redesigned mask and $\mathrm{CO}_{2}$ concentration at trachea, as compared with $6.7 \pm 6.2 \mathrm{~mm} \mathrm{Hg}$ between $P_{\mathrm{ETCO}_{2}}$ measured by nasal cannula and trachea $(P<.001)$. In the human mask fit study, 35 children $(13 \pm 4 \mathrm{~kg})$ with the small mask and $38(24 \pm 8 \mathrm{~kg})$ with the medium mask were evaluated. Capnography tracing was successfully obtained in $86 \%$ of the small and $100 \%$ of the medium masks. In children with small-size masks, the gap between the face mask rim and the child's face was not statistically different among those with good mask fit and without $(1.0 \pm 1.5 \mathrm{~mm}$ vs $1.4 \pm 1.9 \mathrm{~mm}, P=.73)$. CONCLUSIONS: $P_{\mathrm{ETCO}_{2}}$ measurement by a redesigned open-system face mask with a mainstream $\mathrm{CO}_{2}$ detector was accurate in the bench setting. The redesigned face mask can attain good mask fit and accurate capnography tracings in the majority of infants and children. Key words: mask; oxygen; pediatrics; noninvasive; capnography; fit. [Respir Care 2017;62(1):70-77. (c) 2017 Daedalus Enterprises]
\end{abstract}

\section{Introduction}

Capnometry, or partial pressure of end-tidal $\mathrm{CO}_{2}$ $\left(\mathrm{P}_{\mathrm{ETCO}_{2}}\right)$, is considered a standard of care during invasive

\footnotetext{
Ms Napolitano is affiliated with the Respiratory Therapy Department; Drs Nishisaki, Maltese, and Nadkarni are affiliated with the Division of Anesthesiology and Critical Care Medicine; and Ms Buffman, Ms Leffelman, and Dr Nadkarni are affiliated with the Center for Simulation, Advanced Education and Innovation, Children's Hospital of Philadelphia, Philadelphia, Pennsylvania.
}

This study was supported by an unrestricted grant by Nihon-Kohden. Ms Napolitano, Ms Buffman, Ms Leffelman, and Drs Nishisaki and Nadkarni have disclosed a relationship with Nihon Kohden. Ms Napolitano mechanical ventilation in critically ill infants and children. The efficacy of $\mathrm{P}_{\mathrm{ETCO}_{2}}$ is well known for detecting apnea, tracheal tube dislodgement, or ventilation failure. However, it has not been a standard practice for children with a natural airway undergoing sedation despite the current recommendation by the American Society of Anesthesiology. ${ }^{1}$ The capability of $\mathrm{P}_{\mathrm{ETCO}_{2}}$ to detect apnea and hypoventilation is well documented but could be missed when

\footnotetext{
has also disclosed relationships with Draeger Medical, Aerogen, and Respironics. Dr Maltese has disclosed no conflicts of interest.

Ms Napolitano presented a version of this work at the AARC Congress 2015, held November 7-10, 2015, in Tampa, Florida.
} 
a child is receiving supplemental oxygen when monitoring is done by pulse oximetry alone. ${ }^{2-8}$ Several barriers exist for widespread implementation of $\mathrm{P}_{\mathrm{ETCO}}$ monitoring during sedation in clinical practice. They include: (1) lack of face mask options to accurately measure $\mathrm{P}_{\mathrm{ETCO}}$, (2) patients' inability to tolerate nasal cannula, and (3) perceived uncertainty in accuracy of the data. ${ }^{9,10}$

An open-system face mask was developed to deliver oxygen while measuring mainstream $\mathrm{P}_{\mathrm{ETCO}}$ for children in Japan: the cap-ONE mask (Nihon Kohden Corporation, Tokyo, Japan). ${ }^{11}$ However, our local evaluation of this device revealed difficulty in obtaining reliable $\mathrm{P}_{\mathrm{ETCO}_{2}}$ measurement with a larger gap between blood gas $\mathrm{CO}_{2}$ and $\mathrm{P}_{\mathrm{ETCO}_{2}}$ (difference between $\mathrm{P}_{\mathrm{aCO}}$ and $\mathrm{P}_{\mathrm{ETCO}_{2}}: 4.7 \pm 6.9 \mathrm{~mm} \mathrm{Hg}$, $n=14$ ). This was probably due to poor mask fit from the diverse ethnic backgrounds of American children with more depth to their facial features.

The accuracy of $\mathrm{P}_{\mathrm{ETCO}_{2}}$ monitoring is known to be affected by supplemental oxygen flow, tidal volume, and fit of the mask. Our engineer subsequently made substantial design changes to improve mask fit and capture of expiratory gas flow, as detailed below.

The primary objectives of this study were (1) to evaluate the redesigned open-system face mask for $\mathrm{P}_{\mathrm{ETCO}_{2}}$ accuracy using a simulator in a bench setting and (2) to evaluate mask fit and capability to obtain a square $\mathrm{P}_{\mathrm{ETCO}_{2}}$ waveform (accuracy) on infants and children.

\section{Methods}

This study was approved by the institutional review board at the Children's Hospital of Philadelphia. All legal guardians of the children provided written consent to participate in this study procedure.

To improve mask fit, the manufacture engineer made 2 changes to the previous open-system face mask: (1) incorporation of deform-and-hold shaping technology and (2) adjustment of the expiratory gas flow direction cup (Fig. 1). The first change was made to improve the fit of the face mask to children with different facial features. The second change was made to improve efficiency to guide expiratory gas to a capnometer by adopting an anterior-posterior adjustable expiratory gas flow direction cup (gas collection cup) to allow more efficient expiratory gas flow to a small, lightweight (4-g) capnometer (see Fig. 1). Two mask sizes were developed: small for infants weighing 7-20 kg

\footnotetext{
Correspondence: Natalie Napolitano MPH RRT-NPS FAARC, Respiratory Therapy Department, 7NW149, Children's Hospital of Philadelphia, 3401 Civic Center Boulevard, Philadelphia, PA 19104. E-mail: naplitanon@email.chop.edu.
}

DOI: $10.4187 /$ respcare. 04751

\section{QUICK LOOK}

\section{Current knowledge}

Noninvasive partial pressure of end-tidal $\mathrm{CO}_{2}\left(\mathrm{P}_{\mathrm{ETCO}_{2}}\right)$ monitoring improves safety and adverse events during sedation of pediatric patients outside the operating room. Detection of hypopnea, hypoventilation, and apnea is greatly increased with $\mathrm{P}_{\mathrm{ETCO}_{2}}$ compared with assessment alone.

\section{What this paper contributes to our knowledge}

Mainstream capnography was more accurate than sidestream capnography in the spontaneously breathing pediatric bench model. The ability to utilize mainstream noninvasive capnography in pediatric patients receiving sedation outside the operating room has the potential to further improve safety in this population.

and medium for children weighing $10-40 \mathrm{~kg}$. The overlap in patient weight was intentional, to allow providers to choose a face mask with the best fit.

\section{Bench Study}

Simulator Setting. A hybrid manikin lung simulator was developed to evaluate $\mathrm{P}_{\mathrm{ETCO}_{2}}$ accuracy measured by a redesigned open-system face mask with a mainstream capnometer. This simulator mode led a spontaneously ventilating infant and child with a natural airway. The following equipment was used: breathing simulator (ASL5000, IngMar Medical, Pittsburgh, Pennsylvania), infant manikin (Infant Airway Management Trainer, Laerdal, Wappingers Falls, New York), and child airway manikin (AIRSIM CHILD, TruCorp, Belfast, United Kingdom), and a mass flow controller (s-B40/B50, Horiba ESTEC, Kyoto, Japan) (Fig. 2). The breathing simulator, supplying ventilatordriven spontaneous breaths through the infant lung, was connected to the head manikin, and $100 \% \mathrm{CO}_{2}$ gas was blended in via a mass flow controller. Expired $\mathrm{CO}_{2}$ concentration at the trachea was kept at a constant $38 \pm 5 \mathrm{~mm} \mathrm{Hg}$ during the evaluation, which was continuously monitored at the level of trachea by a patient monitor (BSM-6301A/AG-920R, Nihon Kohden, Tokyo, Japan). The expiratory tidal volume and breathing frequency were set to simulate those of a 1-y-old child (frequency, 35 breaths/min; expiratory tidal volume, $115 \mathrm{~mL}$ ) and a 6-y-old child (frequency, 25 breaths/min; expiratory tidal volume, $215 \mathrm{~mL}) .{ }^{12}$

Capnometry Evaluation Procedure. $\mathrm{P}_{\mathrm{ETCO}_{2}}$ measured by the redesigned open-system face mask with a main- 

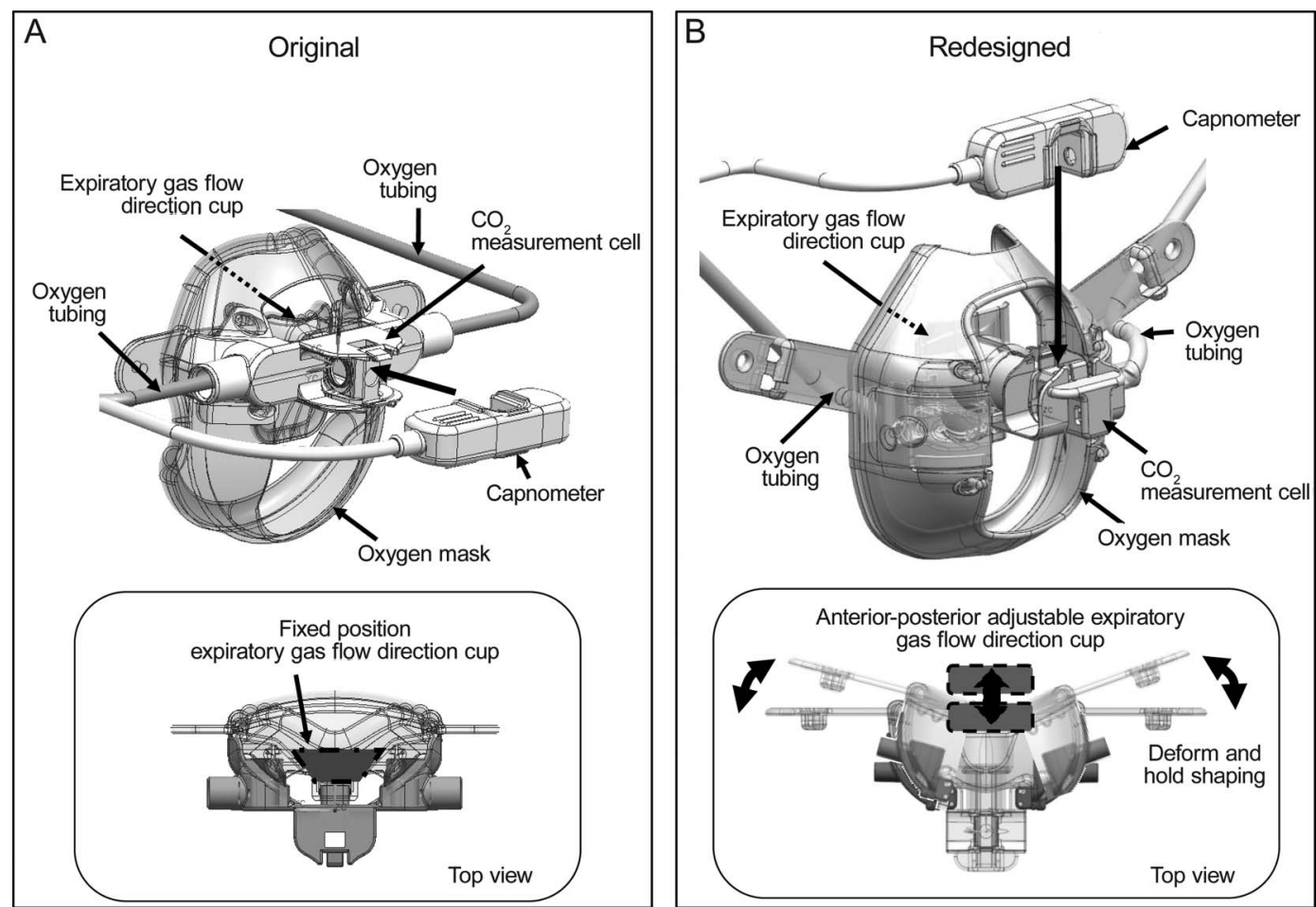

Fig. 1. The changes in the design from the original version of the face mask (A) and the redesigned face mask (B). The capnometer orientation was changed; the expiratory gas flow cup now had anterior-posterior movement with the deform-and-hold shaping of the mask string connections. These design changes allow for a tighter fit for most facial structures, improving the capnography waveform.

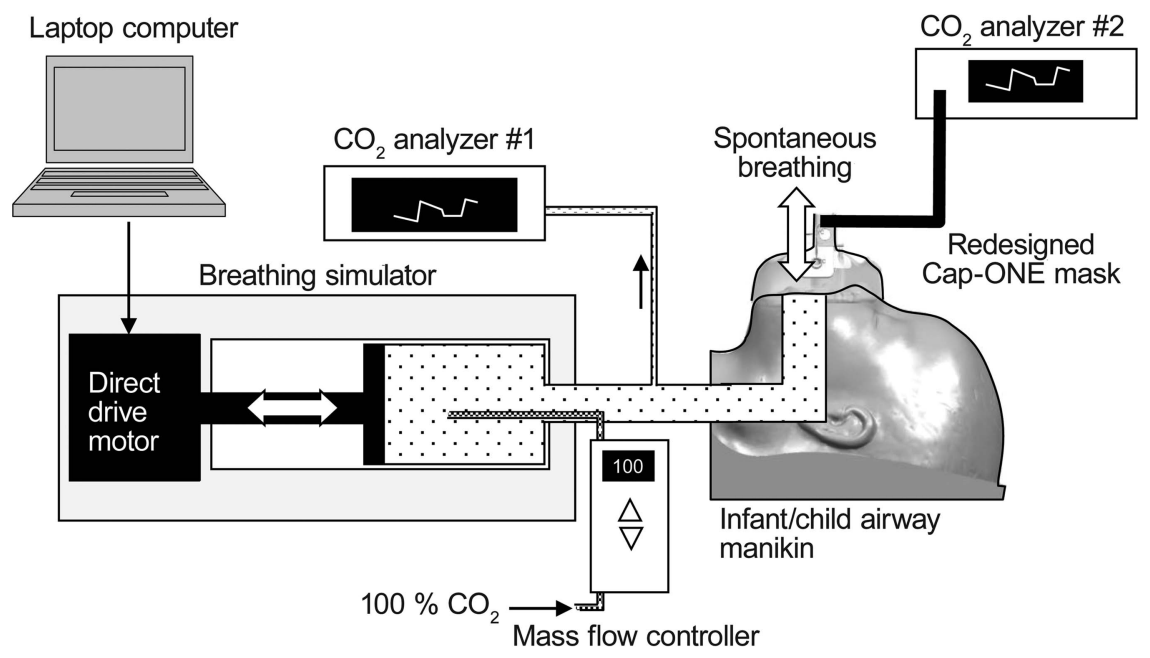

Fig. 2. Spontaneous Breathing Simulator: $\mathrm{CO}_{2}$ analyzer \#1 measures the $\mathrm{CO}_{2}$ directly sampled from the trachea. $\mathrm{CO}_{2}$ analyzer \#2 measures $\mathrm{CO}_{2}$ from the redesigned Cap-ONE mask mainstream capnometer. ASL 5000 was used as an actively breathing patient mode connected to the infant and child manikin heads.

stream capnometer was compared against the $\mathrm{P}_{\mathrm{ETCO}_{2}}$ sampled from a tracheal level for each breath, as shown in Figure 2. Before the start of each experiment, we ensured that the $2 \mathrm{P}_{\mathrm{ETCO}_{2}}$ monitors were both equivalently calibrated to 0 and $38 \mathrm{~mm} \mathrm{Hg}$ using air and $5 \% \mathrm{CO}_{2}$ calibration gas. The $\mathrm{P}_{\mathrm{ETCO}}$ level was recorded at every $25 \mathrm{~ms}$. We also compared the $\mathrm{P}_{\text {ETCO }}$ measured with a commer- cially available and widely used sidestream nasal cannula (pediatric nasal cannula, Salter Labs, Carlsbad, California). Supplemental oxygen was given through the same face mask or nasal cannula used for capnometry. Oxygen flow was applied at $0,0.5,1,2,3,4,5$, and $6 \mathrm{~L} / \mathrm{min}$ with at least a 1-min interval between each flow. The $\mathrm{F}_{\mathrm{IO}_{2}}$ from the trachea was also measured to specifically assess the 


\section{Open-System Oxygen Face Mask With Capnometer}

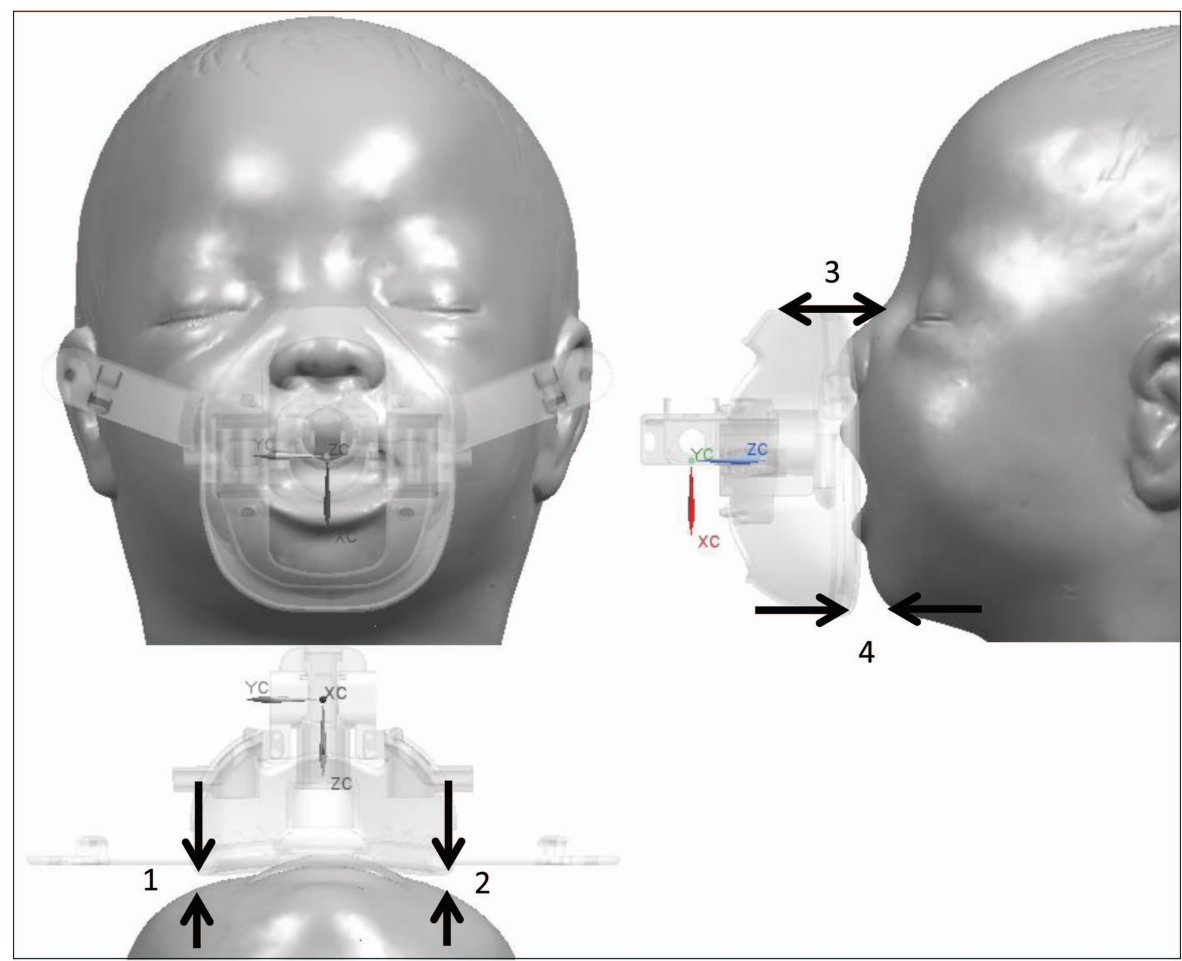

Fig. 3. Manually measured facial gaps. Gaps between the face and the mask that were manually measured during the clinical fit study: (1) gap between right cheek and mask, (2) gap between left cheek and mask, (3) gap between the bridge of the nose and the mask, (4) gap between the chin and the mask.

efficiency for oxygen delivery with face mask and nasal cannula. Spontaneous ventilation was continued for $1 \mathrm{~min}$ at each oxygen flow, and measurements from the last $10 \mathrm{~s}$ were used for analysis.

\section{Clinical Study for Mask Fit}

The clinical fit study took place between March 2014 and January 2015 at a large United States children's hospital and in community centers. The primary location was the preoperative area or the radiology waiting room at a children's hospital. The second location was off site at a church youth group. All locations were approved by the institutional review board, and all subjects went through a standard written consent/assent process. Inclusion criteria were: (1) 7-40-kg body weight, (2) normal cardiovascular and respiratory condition, and (3) no known inherited or acquired facial deformities. After written consent was obtained, a weight-appropriate redesigned open-system face mask (small size for 7-20 kg of body weight, medium size for $10-40 \mathrm{~kg}$ ) was applied to each subject for $30 \mathrm{~s}$, and continuous $\mathrm{P}_{\text {ETCO }}$ waveform was recorded. For children between 10 and $20 \mathrm{~kg}$ of body weight, investigators chose one size of face mask by visual assessment.

Our primary outcome was a good mask fit, defined as the demonstration of a square shape $\mathrm{CO}_{2}$ waveform during expiration. The following study procedures were performed: (1) 3-dimensional digital scan of the subject's face to document facial features (Kinect, Microsoft, Redmond, Washington), (2) placement of one of the appropriate face masks on the face and measurement of gaps in the mask fit at 4 gap spaces (distance between mask and face at nose, chin, left cheek, and right cheek) using a tape measure (Medi-Pak Tape Measure 36, McKesson Health Solutions, Gaithersburg, Maryland) (Fig. 3), and (3) a 30-s $\mathrm{P}_{\mathrm{ETCO}_{2}}$ waveform recording while the gaps were being measured. The data obtained by the 3-dimensional digital facial scan were further analyzed using computer-aided design software (NX7.5, ISID, Tokyo, Japan) to measure predefined facial features (Fig. 4).

\section{Statistical Methods}

The data from all enrolled subjects were analyzed. Demographic characteristics and the gaps between mask and face were summarized by descriptive summaries with means and SD values for continuous variables and percentages for categorical variables. Based on our preliminary data, we hypothesized that each mask (small and medium size) would meet mask fit criteria in $90 \pm 10 \%$ of the subjects. For each mask size, 35 subjects would be required. Considering possible subject withdrawals, we 


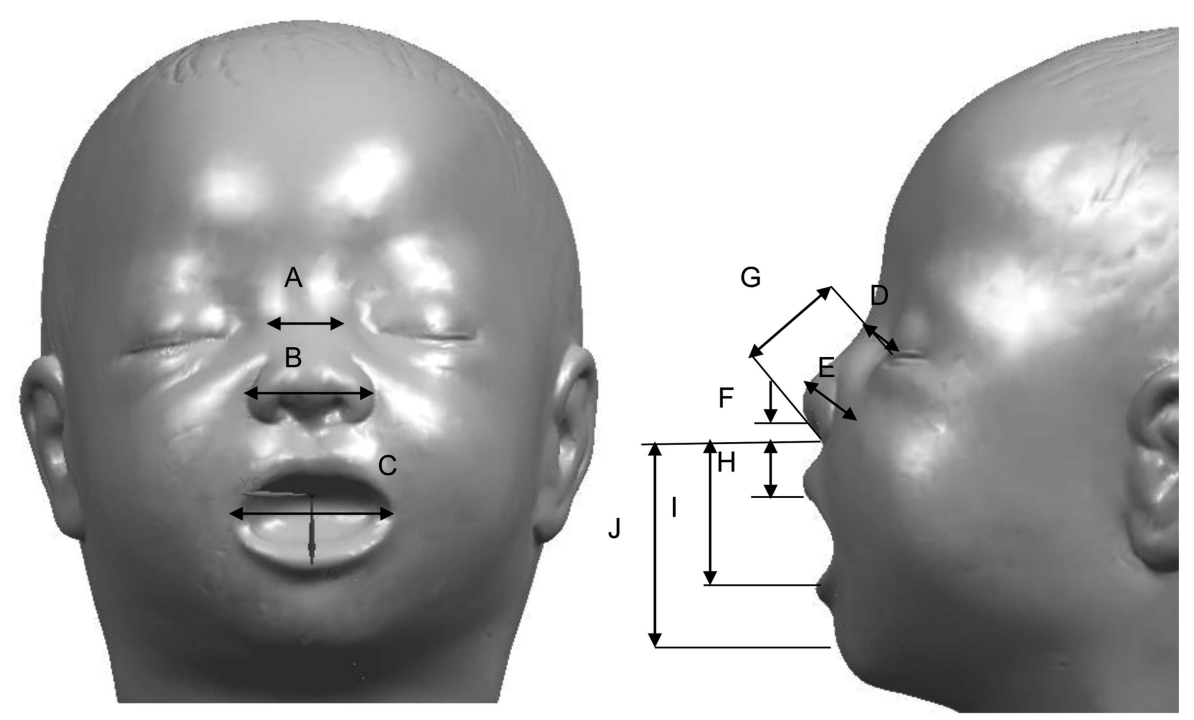

Fig. 4. Anthropometric measurements from 3-dimensional images. Descriptions of the measurements made from the 3-dimensional images of the faces during the clinical fit study: nasal root breadth $(A)$, nasal breadth $(B)$, lip length $(C)$, nose root height $(D)$, nose height $(E)$, nostril height $(F)$, nose length $(G)$, nose to upper lip length $(H)$, nose to lower lip length $(I)$, nose to chin length $(J)$.

planned to recruit 41 subjects ( $15 \%$ dropout rate) for each weight group (a total of 82 subjects).

The Fisher exact test was applied for categorical variables, $t$ test for normally distributed continuous variables, and Wilcoxon rank-sum test for continuous non-normally distributed variables. Normality was evaluated by a histogram. $P<.05$ was used as the criterion for statistical significance. All data were analyzed using Stata 11 (StataCorp, College Station, Texas).

\section{Results}

\section{Bench Study}

$\mathrm{P}_{\mathrm{ETCO}_{2}}$ measurement by open-system face mask was evaluated using a simulator at an $\mathrm{O}_{2}$ flow incrementally increased from 0 to $6 \mathrm{~L} / \mathrm{min}$. The difference between $\mathrm{P}_{\mathrm{ETCO}_{2}}$ measured by face mask and at the level of trachea was significantly smaller than the difference between $\mathrm{P}_{\mathrm{ETCO}}$ measured by nasal cannula and at the level of trachea at 4 , 5 , and $6 \mathrm{~L} / \mathrm{min}$ of $\mathrm{O}_{2}$ flow in both small and medium-size masks (Fig. 5). The $\mathrm{F}_{\mathrm{IO}_{2}}$ delivered to the trachea via the open-system face mask did not exceed 0.50 at high oxygen flow, whereas the nasal cannula proportionally increased $\mathrm{F}_{\mathrm{IO}_{2}}$ (Fig. 6).

\section{Clinical Fit Study}

A total of 73 children were enrolled (35 for the smallsize mask and 38 for the medium-size mask). Enrollment was completed with 73 subjects because we did not experience any dropout. Demographic data and anthropometric
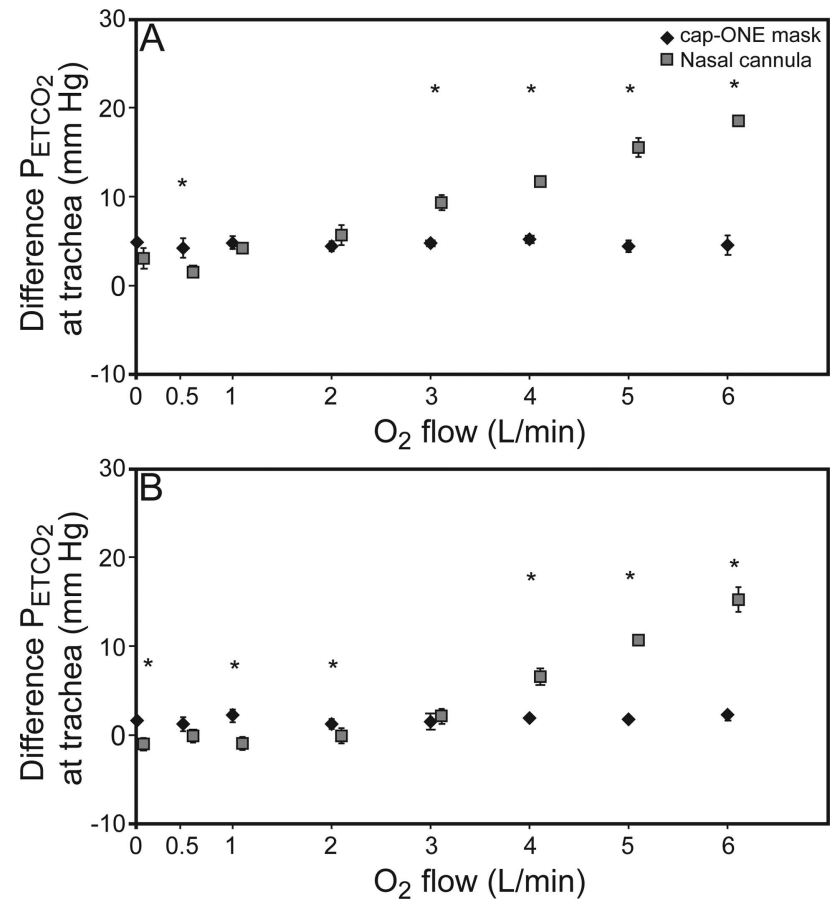

Fig. 5. Bench comparison of nasal cannula and open-system face mask (Cap-ONE) for partial pressure of end-tidal CO2 $\left(\mathrm{P}_{\mathrm{ETCO}_{2}}\right)$ accuracy. Shown is the difference between measured $\mathrm{P}_{\mathrm{ETCO}}$ by open-system face mask, nasal cannula, and lower tracheal $\mathrm{CO}_{2}$ at various mask and cannula oxygen flows. A: small mask size; $B$ : medium mask size. Error bars indicate SD. ${ }^{*} P<.05$.

measures are summarized in Table 1 . The weight of children for the small-size mask was $12.8 \pm 3.7 \mathrm{~kg}$, and for the medium-size mask, it was $23.8 \pm 8.1 \mathrm{~kg}$. Sixty-eight subjects met criteria for good mask fit: 30 of $35(86 \%)$ for 

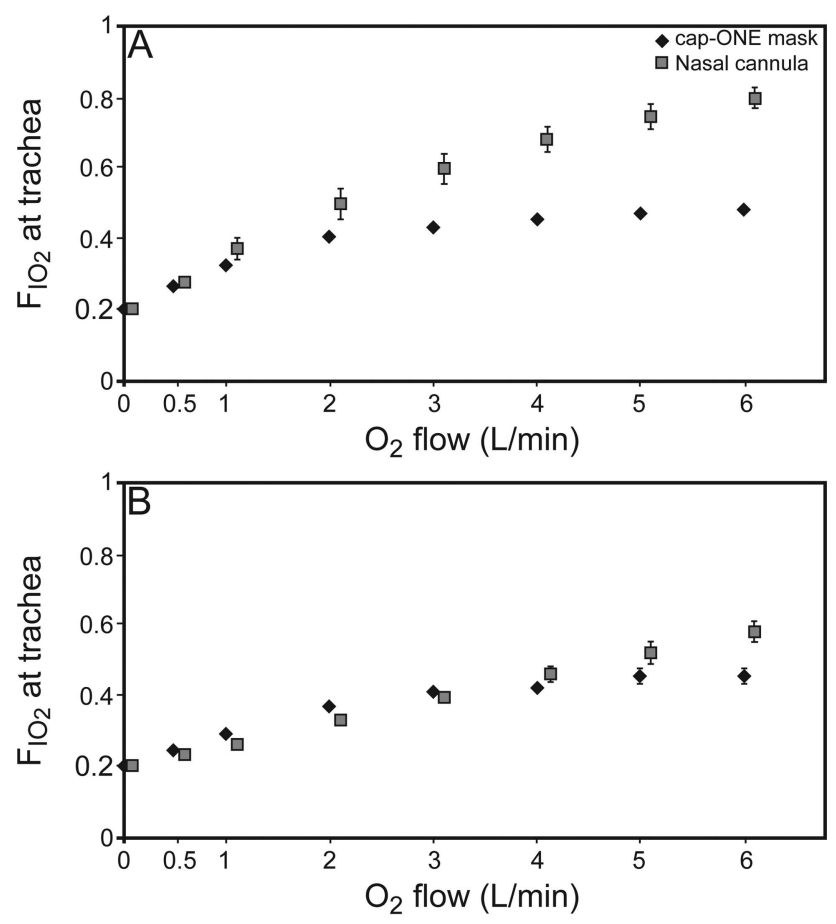

Fig. 6. Comparison of nasal cannula and open-system face mask (Cap-ONE) and $\mathrm{F}_{\mathrm{IO}_{2}}$ accuracy. Difference between $\mathrm{F}_{\mathrm{IO}_{2}}$ by open face mask, nasal cannula, and lower tracheal $\mathrm{F}_{\mathrm{IO}_{2}}$ at various oxygen flows. A: small mask size; B: medium mask size. Error bars indicate SD over the 10 -s sampling time.

Table 1. Clinical Study Subjects Demographic Data With Anthropometric Measures

\begin{tabular}{|c|c|c|}
\hline Variable & $\begin{array}{l}\text { Small-Size Mask } \\
\quad(n=35)\end{array}$ & $\begin{array}{c}\text { Medium-Size Mask } \\
\quad(n=38)\end{array}$ \\
\hline Age, mean \pm SD mo & $28.1 \pm 19.8$ & $75.7 \pm 35.5$ \\
\hline Weight, mean \pm SD kg & $12.8 \pm 3.7$ & $23.8 \pm 8.1$ \\
\hline Female sex, $n(\%)$ & $13(37)$ & $14(37)$ \\
\hline \multicolumn{3}{|l|}{ Race, $n(\%)$} \\
\hline Asian & $1(3)$ & $2(5)$ \\
\hline African-American & $3(8)$ & $7(18)$ \\
\hline Caucasian & $30(86)$ & $28(74)$ \\
\hline Other & $1(3)$ & $1(3)$ \\
\hline \multicolumn{3}{|l|}{$\begin{array}{l}\text { Anthropometric measurements, } \\
\text { mean } \pm \text { SD mm* }\end{array}$} \\
\hline A. Nasal root breadth & $18.6 \pm 2.8$ & $20.8 \pm 2.6$ \\
\hline B. Nose breadth & $27.8 \pm 4.1$ & $30.4 \pm 4.8$ \\
\hline C. Lip length & $42.0 \pm 5.5$ & $48.2 \pm 6.9$ \\
\hline D. Nose root height & $7.2 \pm 1.3$ & $9.0 \pm 2.2$ \\
\hline E. Nose height & $13.9 \pm 3.0$ & $16.7 \pm 3.0$ \\
\hline F. Nostril height & $6.5 \pm 1.1$ & $7.8 \pm 1.4$ \\
\hline G. Nose length & $41.7 \pm 5.0$ & $46.1 \pm 5.3$ \\
\hline H. Nose to upper lip length & $11.3 \pm 1.7$ & $12.4 \pm 2.4$ \\
\hline I. Nose to lower lip length & $24.1 \pm 6.0$ & $24.7 \pm 4.3$ \\
\hline J. Nose to chin length & $55.7 \pm 8.2$ & $60.9 \pm 6.2$ \\
\hline
\end{tabular}

Table 2. Gaps Between Mask and Face Measured by 3-Dimensional Scanning Technology

\begin{tabular}{lccc}
\hline \hline \multicolumn{1}{c}{ Gap Measurement } & $\begin{array}{c}\text { Small-Size } \\
\text { Mask }(n=33)\end{array}$ & $\begin{array}{c}\text { Medium-Size } \\
\text { Mask }(n=34)\end{array}$ & $P$ \\
\hline Right cheek and mask, mm & $0.1 \pm 0.5$ & $0.1 \pm 0.4$ & .98 \\
Left cheek and mask, mm & $0.3 \pm 1.2$ & $0.1 \pm 0.4$ & .38 \\
Nose and mask, mm & $4.8 \pm 7.1$ & $11.2 \pm 5.9$ & $<.001$ \\
Chin and mask, mm & $0.2 \pm 0.5$ & $0.8 \pm 1.8$ & .045
\end{tabular}

Results are mean $\pm \mathrm{SD}$

Gaps were unable to be measured for 2 subjects for the small mask and 4 subjects for the medium mask.

the small-size mask and 38 of $38(100 \%)$ for the mediumsize mask $(P=.02$, Fisher exact test). Among those children with good fit by $\mathrm{P}_{\mathrm{ETCO}_{2}}$ waveform, the measured $\mathrm{P}_{\mathrm{ETCO}_{2}}$ was $40.1 \pm 6.4$ for the small mask and $38.9 \pm 4.9$ for the medium mask $(P=.20, t$ test). The measured gaps for both small and medium masks are presented in Table 2.

\section{Population Without Adequate $\mathbf{P}_{\mathrm{ETCO}_{2}}$ Waveform}

Adequate $\mathrm{P}_{\text {ETCO }}$ waveform was not obtained in 5 children with the small mask (mean weight $12.7 \pm 3.3 \mathrm{~kg}$, not different from children with adequate waveform, $P=.99$ ). All children with the medium-size mask met the criteria for good mask fit by adequate $\mathrm{P}_{\mathrm{ETCO}_{2}}$ waveforms. All but one child without adequate $\mathrm{P}_{\mathrm{ETCO}_{2}}$ waveform had the highest $\mathrm{P}_{\mathrm{ETCO}_{2}}<20 \mathrm{~mm} \mathrm{Hg}$, confirming poor exhaled gas capture by a mask. In contrast, all children with adequate waveform had $\mathrm{P}_{\mathrm{ETCO}_{2}}>25 \mathrm{~mm} \mathrm{Hg}$. In children with smallsize masks, the gap between face mask rim and the child's face was not statistically different among those with good mask fit and without (average gap: $1.0 \pm 1.5 \mathrm{~mm}$ vs $1.4 \pm 1.9 \mathrm{~mm}, P=.73$, Wilcoxon rank-sum test). These 5 children without good mask fit were primarily mouthbreathing, and the oral collection cap was not big enough to capture the expired air flow from the child's mouth. The oral cup placement reached the top of the upper lip on all of these children.

\section{Discussion}

Our study demonstrated the accuracy of the redesigned open-system face mask with $\mathrm{P}_{\mathrm{ETCO}_{2}}$ measurement in a laboratory setting. Despite the continuous oxygen flow provided at various flows, $\mathrm{P}_{\mathrm{ETCO}_{2}}$ measurement by the open-system face mask remained accurate with a small difference from the sampled gas at the level of the trachea. In contrast, the $\mathrm{P}_{\mathrm{ETCO}_{2}}$ measures by a currently available sidestream nasal cannula system revealed a large difference from the gas sampled at the tracheal level. This dif- 
ference was even larger when the supplemental $\mathrm{O}_{2}$ flow was higher. Differences in waveforms were also observed: The observed $\mathrm{P}_{\mathrm{ETCO}_{2}}$ waveform was crisper in distinguishing inspiratory and expiratory phases of the respiratory cycle with a distinct square form than with the sidestream waveform. There was also a 3.2-s delay seen in measurement for the sidestream nasal cannula compared with the mainstream mask measurement that had immediate response with each breath. The $\mathrm{F}_{\mathrm{IO}_{2}}$ delivered to the trachea via the open-system face mask did not exceed 0.50 at high oxygen flow, whereas the nasal cannula proportionally increased $\mathrm{F}_{\mathrm{IO}_{2}}$ (Fig. 6). This may be due to the difference in oxygen delivery system between 2 devices. Specifically, the open-system face mask allows entrapment of room air freely into the mask before the oxygen-mixed gas is being inhaled by children. In contrast, the nasal cannula directly delivers the gas with high oxygen fraction into the nasal cavity.

Our human mask fit study demonstrated that the redesigned open-system face mask is feasible to measure $\mathrm{P}_{\mathrm{ETCO}}$ accurately in $>90 \%$ of children, even when a range of supplemental $\mathrm{O}_{2}$ flows of $0-6 \mathrm{~L} / \mathrm{min}$ are provided. In 5 children who were mouth breathers, good mask fit was difficult to achieve with the small mask, mainly because the structure to capture expiratory gas flow was located at the upper lip. This problem occurred only in a small portion of smaller children, and we were able to achieve good mask fit with $86 \%$ of this population. Anthropometric measurement and manually measured gap between face mask and face were not different between children with good fit and without good fit based on capnography tracing.

The use of capnography during sedation outside the operating room has been associated with lower incidence of apnea and desaturation in adult patients. ${ }^{13,14} \mathrm{~A}$ metaanalysis in adult subjects who received procedural sedation showed that capnography use during the sedation was 17.6 times (95\% CI 2.5-122.1, $P=.004$ ) more likely to detect apnea. ${ }^{13}$ Langhan et al ${ }^{15}$ performed a randomized clinical study in pediatric subjects requiring sedation. They demonstrated that persistent hypoventilation was less often observed in the intervention group than in the control group (0.004 vs 0.010 events/subject min of sedation, $P=.004)$. Lightdale et a ${ }^{16}$ performed a randomized, controlled trial undergoing moderate sedation for endoscopy in children. The intervention group utilized a nasal cannula sidestream capnometer (Microstream system; Oridion Medical, Needham, Massachusetts) with a manual alarm by study personnel at the time of 15-s hypoventilation/apnea, in addition to a standard continuous monitoring with electrocardiogram and pulse oximetry. The intervention group had an $11 \%$ incidence of hypoxemia, significantly lower than the control group $(25 \%, P=.02)$. A similar study was replicated by Qadeer et al ${ }^{17}$ in adult subjects undergoing elective endoscopic retrograde cholangiopancreatog- raphy or endoscopic ultrasonography under procedural sedation.

The American Society of Anesthesiologists currently recommends the use of capnometry for pediatric patients receiving sedation with a natural airway. ${ }^{1}$ However, our study demonstrated that the sidestream $\mathrm{P}_{\mathrm{ETCO}_{2}}$ measurement by nasal cannula is less accurate when compared with a face mask mainstream capnometry system. This results in underestimating high $\mathrm{P}_{\mathrm{ETCO}_{2}}$ from hypopnea or overdiagnosing low $\mathrm{P}_{\mathrm{ETCO}_{2}}$. The mainstream $\mathrm{P}_{\mathrm{ETCO}_{2}}$ measurement may be associated with a more accurate distinct square-shaped waveform and shorter response time to the change of $\mathrm{P}_{\mathrm{ETCO}}$. Therefore, the tested open-system face mask with a mainstream $\mathrm{P}_{\mathrm{ETCO}}$ measurement device seems a promising solution, especially when supplemental $\mathrm{O}_{2}$ is provided simultaneously.

We attempted to explore the association between our clinical criteria of mask fit with appropriate $\mathrm{P}_{\mathrm{ETCO}}$ tracing with anatomical facial features or externally measured gaps between mask rims and facial surface. Fit testing of masks to ensure accuracy of gas delivery or measurements has been traditionally performed in the aviation field. Schreinemakers et al ${ }^{18}$ documented the facial fit of oxygen masks used by F-16 pilots to determine reasons for nasal discomfort and injury. They utilized 3-dimensional imaging of the face with and without the mask to determine contact points and angle of the face to determine an appropriate fit for pilots, similar to our study measurements. Using 3-dimensional imaging data, they further redesigned the mask to improve the fit of the mask to pilots. Our study was not able to detect distinct differences between children with good mask fit and without good mask fit (poor $\mathrm{P}_{\mathrm{ETCO}_{2}}$ tracing). This may be due to the specific measurement we chose in this study. All children without good mask fit were mouth breathers, and research staff were able to identify the cause of failure (ie, the position of the expiratory flow cup being at the upper lip). Future studies are needed using the 3-dimensional scanning technology to improve a mask fit with simulation utilizing 3-dimensional printing technology.

\section{Limitations}

This study has some important limitations. First, the mask fit criteria were based on the capnography waveform, not the absolute $\mathrm{P}_{\mathrm{ETCO}}$ measurement. However, in our analysis, we identified no subjects in the good mask fit group with a $\mathrm{P}_{\mathrm{ETCO}_{2}}<25 \mathrm{~mm} \mathrm{Hg}$. For practical reasons, we did not attempt to demonstrate the accuracy of $\mathrm{P}_{\mathrm{ETCO}_{2}}$ measurement in children compared with invasive arterial blood gas values. The $\mathrm{F}_{\mathrm{IO}_{2}}$ at the tracheal level was lower in the open-system face mask than the nasal cannula at all tested oxygen flows. However, the open-system face mask was able to achieve $\mathrm{F}_{\mathrm{IO}_{2}}$ oxygen delivery $>0.40$ without 


\section{Open-System Oxygen Face Mask With Capnometer}

attenuating $\mathrm{P}_{\mathrm{ETCO}_{2}}$ measurement accuracy, which is clinically sufficient.

\section{Conclusions}

$\mathrm{P}_{\mathrm{ETCO}_{2}}$ measurement by a deform-and-hold shaping technology and anterior-posterior adjusting expiratory gas flow cup redesigned open-system face mask with a mainstream $\mathrm{CO}_{2}$ detector was accurate in the bench setting. The redesigned face mask can attain good mask fit and accurate capnography tracings in the majority of infants and children.

\section{REFERENCES}

1. American Academy of Pediatrics, American Academy of Pediatric Dentistry, Coté C, Wilson S. Guidelines for monitoring and management of pediatric patients during and after sedation for diagnostic and therapeutic procedures: an update. Pediatrics 2006;118(6):25872602.

2. Poirer MP, Gonzalez Del-Rey JA, McAneney CM, DiGiulio GA. Utility of monitoring capnography, pulse oximetry and vital signs in the detection of airway mishaps: a hyperoxemic animal model. Am J Emerg Med 1998;16(4):350-352.

3. Burton JH, Harrah JD, Germann CA, Dillon DC. Does end-tidal carbon dioxide monitoring detect respiratory events prior to current sedation monitoring practices? Acad Emerg Med 2006;13(5):500504.

4. Patel R, Lenczyk M, Hannallah RS, McGill WA. Age and the onset of desaturation in apneic children. Can J Anaesth 1994;41(9):771774.

5. Fu ES, Downs JB, Schweiger JW, Miguel RV, Smith RA, Supplemental oxygen impairs detection of hypoventilation by pulse oximetry. Chest 2004;126(5):1552-1558.

6. Kinouchi K, Fukumitsu K, Tashiro C, Takauchi Y, Ohashi Y, Nishida $\mathrm{T}$. Duration of apnoea in anaesthetized children required for desaturation of haemoglobin to $95 \%$ : comparison of three different breathing gases. Paediatr Anaesth 1995;5(2):115-119.
7. Farmery AD, Roe PG, A model to describe the rate of oxyhaemoglobin desaturation during apnoea. Br J Anaesth 1996;76(2):284291

8. Verhoeff F, Sykes MK. Delayed detection of hypoxic events by pulse oximeters: computer simulations. Anaesthesia 1990;45(2):103109

9. Iyer NS, Koziel JR, Langhan ML. A qualitative evaluation of capnography use in paediatric sedation: perceptions, practice and barriers. J Clin Nurs 2015;24(15):2231-2238.

10. Langhan ML, Kurtz JC, Schaeffer P, Asnes AG, Riera A. Experiences with capnography in acute care settings: a mixed-methods analysis of clinical staff. J Crit Care 2014;29(6):1035-1040.

11. Takatori F, Yamamori S, Inoue M, Abe S, Miyasaka K. A novel mainstream capnometer system for non-intubated pediatric patients requiring oxygen administration. Conf Proc IEEE Eng Med Biol Soc 2011;2011:1189-1192.

12. Walsh BK. Neonatal and Pediatric Respiratory Care, 4th edition. St Louis: Elsevier; 2015:54, 81.

13. Waugh JB, Epps CA, Khodneva YA. Capnography enhances surveillance of respiratory events during procedural sedation: a metaanalysis. J Clin Anesth 2011;23(3):189-196.

14. Vargo JJ, Zuccaro G Jr., Dumot JA, Conwell DL, Morrow JB, Shay SS. Automated graphic assessment of respiratory activity is superior to pulse oximetry and visual assessment for the detection of early respiratory depression during therapeutic upper endoscopy. Gastrointest Endosc 2002;55(7):826-831

15. Langhan ML, Shabanova V, Li FY, Bernstein SL, Shapiro ED. A randomized controlled trial of capnography during sedation in a pediatric emergency setting. Am J Emerg Med 2015;33(1):25-30.

16. Lightdale JR, Goldmann DA, Feldman HA, Newburg AR, DiNardo JA, Fox VL. Microstream capnography improves patient monitoring during moderate sedation: a randomized, controlled trial. Pediatrics 2006;117(6):e1170-e1178.

17. Qadeer MA, Vargo JJ, Dumot JA, Lopez R, Trolli PA, Stevens T, et al. Capnographic monitoring of respiratory activity improves safety of sedation for endoscopic cholangiopancreatography and ultrasonography. Gastroenterology 2009;136(5):1568-1576.

18. Schreinemakers JR, Oudenhuijzen AJ, van Amerongen PC, Kon M. Oxygen mask fit analysis in F-16 fighter pilots using 3D imaging. Aviat Space Environ Med 2013;84(10):1029-1033. 